\title{
Secondary antinuclei from supernova remnants and background for dark matter searches
}

\author{
Nicola Tomassetti*i \\ Department of Physics and Earths Science, Università di Perugia, and INFN-Perugia, I-06100 \\ Perugia, Italy
}

\author{
Alberto Oliva \\ Centro de Investigaciones Energéticas, Medioambientales y Tecnológicas CIEMAT, E-28040 \\ Madrid, Spain
}

\begin{abstract}
We compute the spectra of cosmic-ray (CR) nuclei and anti-nuclei under a scenario where hadronic interaction processes inside supernova remnants (SNRs) can produce a diffusivelyshock-accelerated "source component" of secondary particles. This scenario is able to explain the recent measurements reported by AMS on the antiproton/proton ratio, that is found to be remarkably constant at $\sim 60-450 \mathrm{GeV}$ of kinetic energy. However, as we will show, this explanation is ruled out by the new AMS data on the $\mathrm{B} / \mathrm{C}$ ratio, which is found to decrease steadily up to $\mathrm{TeV} / \mathrm{n}$ energies. With the constraints provided by the two ratios, we calculate conservative (B/C driven) and speculative ( $\bar{p} / p$ driven) SNR-induced flux contribution for the spectra of antideuteron and antihelium in CRs, along with their standard secondary component expected from CR collisions in the interstellar gas. We found that the SNR component of anti-nuclei can be significantly large at high-energy, above a few $\sim 10 \mathrm{GeV} / \mathrm{n}$, but it is always sub-dominant at sub-GeV/n energies, that is, the energy region where dark-matter induced signals may exceed the standard astrophysical background. Furthermore, the total antinuclei flux from insterstellar spallation plus SNR-component is tightly bounded by the data, so that hadronic production in SNRs has a minor impact on the astrophysical background for dark matter searches.
\end{abstract}

35th International Cosmic Ray Conference - ICRC2017 -

10-20 July, 2017

Bexco, Busan, Korea

\footnotetext{
${ }^{*}$ Speaker.

$\dagger$ E-mail: nicola.tomassetti@cern.ch

†E-mail: alberto.oliva@cern.ch
} 


\section{Introduction}

Light antinuclei in cosmic rays (CRs) such as antiproton $(\bar{p})$, antideuteron $(\overline{\mathrm{d}})$, and antihelium $(\overline{\mathrm{He}})$ are excellent messengers for the search of dark-matter (DM) in the Galaxy. For a wide range of DM mass, the annihilation of decay of DM particles may generate antinucleons, $\bar{p}$ or $\bar{n}$, which can merge into antinuclei $\left(\overline{\mathrm{d}}, \overline{{ }^{3} \mathrm{H}}, \overline{{ }^{3} \mathrm{He}}\right)$ thereby providing signatures in the energy window below a few $\mathrm{GeV}[1,2,3]$. The antiproton-to-proton $(\bar{p} / p)$ ratio in CRs has been measured by the Alpha Magnetic Spectrometer (AMS) experiment from $\sim 0.5$ to $450 \mathrm{GeV}$ of kineti cenergy [4]. At $E \gtrsim 60 \mathrm{GeV}$, the $\bar{p} / p$ ratio is found to be unexpectedly constant with energy, in contrast to standardmodel calculations of secondary $\bar{p}$ production in the interstellar matter (ISM), from which the $\bar{p} / p$ ratio is expected to decrease with energy. Due to this tension, several authors claimed the need of assessing the astrophysical antimatter background [5, 6, 7, 8, 9]. While interpretations in terms of DM have been proposed [10], it was also suggested that a high-energy antiproton "excess" may arise from hadronic interaction processes inside supernova remnants (SNRs) [11, 12]. A constant $\bar{p} / p$ ratio can in fact arise from a source antiproton component. Calculations in this direction have been performed recently $[13,14,15]$. In contrast, $\overline{\mathrm{d}}$ or $\overline{\mathrm{He}}$ nuclei have never been observed in CRs, yet detection experiments have established tight upper limits to the flux of these particles [1]. The search for antinuclei in CRs is progressing rapidly: AMS is gradually approaching the expected level of astrophysical background [16], while the first science flight of the GAPS project will be held in the coming years [17]. Recently, hints for $\overline{\mathrm{He}}$ events may have been identified by AMS, strengthening the idea of a possible antimatter excess in CRs [18]. Hence the first observation of antinuclei in CRs has concrete chances to be achieved by the incoming generation of CR detection experiments.

In this paper, we present flux calculations of CR nuclei and antinuclei under a model of acceleration and propagation which accounts for the production of secondary particles inside SNRs. Our calculations are carried out within the linear diffusive-shock-acceleration (DSA) theory and the two-halo model of diffusive propagation. Our statistical analysis are based on standard $\chi^{2}$ techniques using new experimental data on the boron-to-carbon $(\mathrm{B} / \mathrm{C})$ and $\bar{p} / p$ ratios released by AMS in the GeV-TeV energy region. As we will show, the two ratios support well the occurrence of secondary production processes inside SNRs. However, B/C-driven and $\bar{p}$-driven constraints give inconsistent results in terms of model parameters describing production and acceleration of CR particles. Using both ratios separately in order to calibrate the model, we derive conservative (i.e., B/C-driven) and speculative $(\bar{p} / p$-driven) predictions for the $\overline{\mathrm{d}}$ and $\overline{\mathrm{He}}$ fluxes in CRs. We discuss our results in the context of dark matter searches in space.

\section{Calculations}

Secondary production calculations involve a large number of CR+ISM reactions, describing the generation of secondary particles and antiparticles from the fragmentation of CR nuclei off the gas (interstellar or circumstellar) composed by hydrogen and helium [19]. We account for the production of several isotopes such as ${ }^{2} \mathrm{H},{ }^{3} \mathrm{He},{ }^{6,7} \mathrm{Li},{ }^{7,9,10} \mathrm{Be}$, and ${ }^{10,11} \mathrm{~B}$ from the disintegration of $\mathrm{C}-\mathrm{N}-\mathrm{O}, \mathrm{Si}$, and $\mathrm{Fe}$, using cross-section formulae evaluated in $[20,13]$ (for the production of $\mathrm{H}-\mathrm{He}-$ isotopes) and in [21] (for Li-Be-B isotopes). We therefore account for production of antinuclei such 
as $\bar{p}, \bar{n}, \overline{\mathrm{d}}, \overline{\mathrm{t}}$ and $\overline{\mathrm{He}}$ from fragmentation of CR proton and helium. The production of antinucleons is modeled using the semi-analytical parameterization of [22], which gives the Lorentz-invariant distribution function, $f_{\mathrm{ISM}}^{C R \rightarrow p, n} \equiv E_{\bar{p}} \frac{d^{3} \sigma}{d p_{p, n}^{3}}$, for all relevant $p-p$ collisions. Dedicated parameterizations [23] were used to handle other CR+ISM collisions ( $p$-He, He- $p, \mathrm{He}-\mathrm{He}$ ). The production of antinuclei such as $\overline{\mathrm{d}}=\{\bar{n}, \bar{p}\}$ and $\overline{\mathrm{He}}=\{\bar{n}, \bar{p}, \bar{p}\}$ has been re-evaluated within an improved formulation of the nuclear coalescence model, based on [25]. According to this model, when $\bar{p}$ or $\bar{n}$ are produced in hadronic jets of nucleus-nucleus collisions, an antinucleus is formed when the relative momenta of all pairs of antinucleons lie within the coalescence momentum $p_{0}$. Using accelerator data [24], we set $p_{0} \cong 90 \mathrm{MeV} / \mathrm{c}$, The production of $\overline{\mathrm{t}}=\{\bar{n}, \bar{n}, \bar{p}\}$ is also included in our calculations of the total $\overline{\mathrm{He}}$ flux. Destruction reaction and tertiary productions are accounted as well. The CR acceleration spectrum is calculated using the linear diffusive-shock-acceleration (DSA) theory, where we account for production and destruction processes of secondary and tertiary fragments. Similar calculations are done in earlier works for CR nuclei [12, 28, 29] and antiprotons [11, 14]. Here we have followed the formalism in [29] as generalized in [13] and now extended to DSA acceleration of antinuclei. The amount of secondary production during acceleration is regulated by the product $\tau_{\text {snr }} n_{-}$between SNR age and SNR gas density; The subsequent propagation of CRs in the ISM is described under a two-halo model of CR diffusion and nuclear interactions [30, 31]. The size of the two halos is defined by $l=0.5 \mathrm{kpc}$ and $L=5 \mathrm{kpc}$. The rigidity $(R)$ dependent diffusion coefficient in proximity of the disk $(|z|<l)$ is $K \propto \beta K_{0}(R / \mathrm{GV})^{\delta_{0}}$. We consider a Kolmogorov-type turbulence spectrum in proximity of the Galactic disk (with $\delta_{0}=1 / 3$ ) where the turbulence is expected to be injected by SNRs. Away from the disk $(l<|z|<L)$, the diffusion scaling index is taken as $\delta_{0}+\Delta$, reflecting the effects of CR-driven turbulent motion. We use value $\Delta=0.55$ which is inferred from primary CR spectra [8]. The total production of secondaries in the ISM depends on the ratio $L / K_{0}$, which is taken as free parameter. The near-Earth CR fluxes are affected by solar modulation. We adopt the force-field approximation using $\phi_{\text {Fisk }}=0.7 \mathrm{GV}$ as modulation potential for AMS [32]. This value was derived using CR proton data. Modulation of antinuclei can be different due to charge-sign dependent effects arising from drift motion. We have investigated this difference $a$ posteriori using a 2D numerical model [33]. However, we found no appreciable difference for the AMS data, because these data are collected across the 2013 polarity reversal, and thus they contain CR particles propagated under both polarity states.

\section{Results: $\mathbf{B} / \mathbf{C}$ and $\bar{p} / p$ ratios}

We use the AMS data on the $\mathrm{B} / \mathrm{C}$ and $\bar{p} / p$ ratios to constrain key SNR parameters: the product $\tau_{\text {snr }} n_{-}$between SNR age and upstream gas density, the ratio $K_{0} / L$ between normalization of diffusion coefficient and half-height of the halo, and the normalization of the diffusion coefficient $D$ at the shock. Fits are performed by means of a standard $\chi^{2}$ analysis, where $\mathrm{B} / \mathrm{C}$ and $\bar{p} / p$ data are used separately. The $\chi^{2}$-estimator is calculated above the minimal energy threshold of $10 \mathrm{GeV} / \mathrm{n}$ in order to prevent possible biases from solar modulation. Other details will be provided in a forthcoming paper. The best-fit models are shown in Fig. 1 for the $\mathrm{B} / \mathrm{C}$ (a) and $\bar{p} / p$ (b) ratio calculations along with the ISM and SNR contributions. As seen from the figure, we have obtained very good fits for both observables, i.e., accounting for secondary production inside SNRs improves the description of the data significantly. The decrease of the ISM-induced $\mathrm{B} / \mathrm{C}$ ratio is well balanced by 

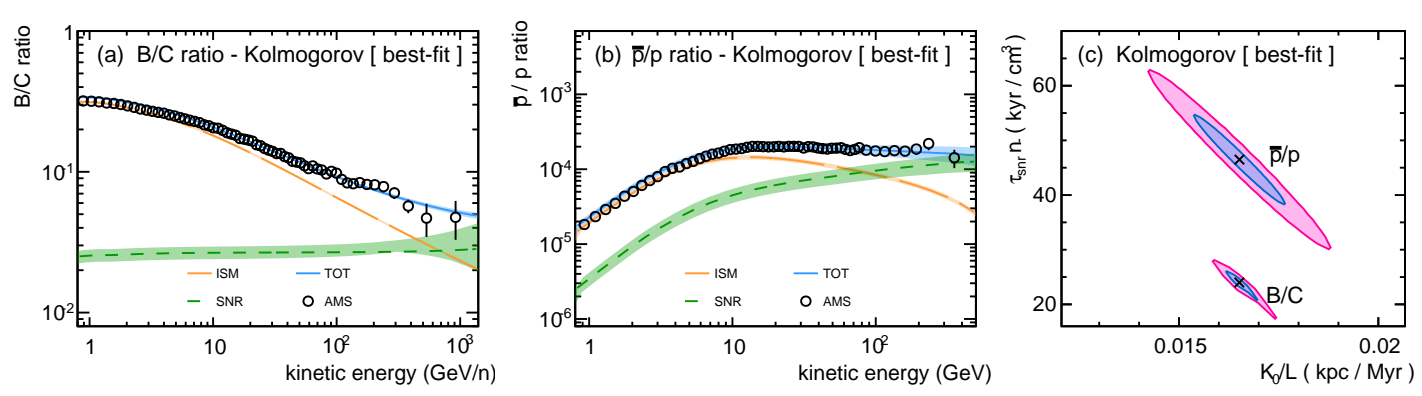

Figure 1: B/C ratio (a) and $\bar{p} / p$ ratio (b) measured by AMS $[34,4]$ in comparison with best-fit models (light blue solid lines) with $E_{\min }=10 \mathrm{GeV} / \mathrm{n}$. Contributing components from standard secondary production (orange, long-dashed lines) are shown together with the component from secondary production in SNRs (green short-dashed lines). The shaded bands represent the errors from the fits. Two-dimensional contour plots (c) are shown for the parameters $\tau_{\mathrm{snr}} n_{-}$and $K_{0} / L$ at $68 \%$ (shaded blue area) and $95 \%$ (shaded pink area) of confidence levels. The plots correspond to B/C-driven (a) and $\bar{p} / p$-driven (b) fits.

the hard SNR-accelerated B-component. Furthermore, tor SNRs of typical age $\tau_{\text {snr }} \cong 20 \mathrm{kyr}$, the best-fit density is close to the average ISM density $\tilde{n} \approx 1 \mathrm{~cm}^{-3}$. Unfortunately, the manifestation of the SNR component at high-energy does not provide striking signatures on the $\mathrm{B} / \mathrm{C}$ ratio, but only a smooth hardening. Furthermore, accounting for SNR-induced contributions in secondary CR fluxes introduces strong degeneracies between the source and transport parameters, as discussed in $[29,36]$ using pre-AMS data. It was also noted that disregarding interactions in SNRs would favor models with weaker dependence for the Galactic diffusion coefficient.

Best-fit models for the $\bar{p} / p$ ratio are shown in Fig. 1(b). It can be noted that this ratio leads to a stronger secondary production in SNRs. Nonetheless, B/C-driven and $\bar{p} / p$-driven fits lead to different parameter values. This can be seen in Fig. 1(c), which shows the $68 \%$ and the $95 \%$ contours for the parameters $K_{0} / L$ and $\tau_{\mathrm{snr}} n_{-}$. The best-fit associated with the two observables lie in separate regions of the parameter space and, in particular, the $\bar{p} / p$ ratio favors denser media for the SNR background plasma. This reflects the fact that, while the $\mathrm{B} / \mathrm{C}$ data decreases steadily up to $E \sim 1 \mathrm{TeV} / \mathrm{n}$, the $\bar{p} / p$ ratio is essentially constant at $E \gtrsim 50 \mathrm{GeV}$. This tension, that was noted under conventional models of CR propagation, is not resolved after accounting for secondary CR production and acceleration inside SNRs.

\section{Results: $\overline{\mathrm{d}}$ and $\overline{\mathrm{He}}$ fluxes}

We now present our model predictions of antideuteron and antihelium energy spectra, where acceleration and propagation parameters are constrained by the AMS data on $\mathrm{B} / \mathrm{C}$ and $\bar{p} / p$ ratios. The results are presented in Fig. 2 for the $\overline{\mathrm{d}}$ and $\overline{\mathrm{He}}$ fluxes near-Earth from the B/C-driven parameter setting of Sect.3. The $\overline{\mathrm{d}}$-fluxes are shown in the left panels and the $\overline{\mathrm{He}}$-fluxes in the right ones. Colors and line styles are encoded as in Fig. 1 so that, along with the standard secondary ISMinduced component (orange), the flux contribution from SNR-accelerated nuclei (green) is shown. The shapes and the intensities of the fluxes are in good agreement with those reported in early works [25]. In particular, all particle fluxes show a characteristic peak at a few $\mathrm{GeV} / \mathrm{n}$ of kinetic energy preceded and followed by quick spectral drops at lower and higher energies. This shape reflects the kinematics of their production and the rapid power-law falling flux of the progenitors, 

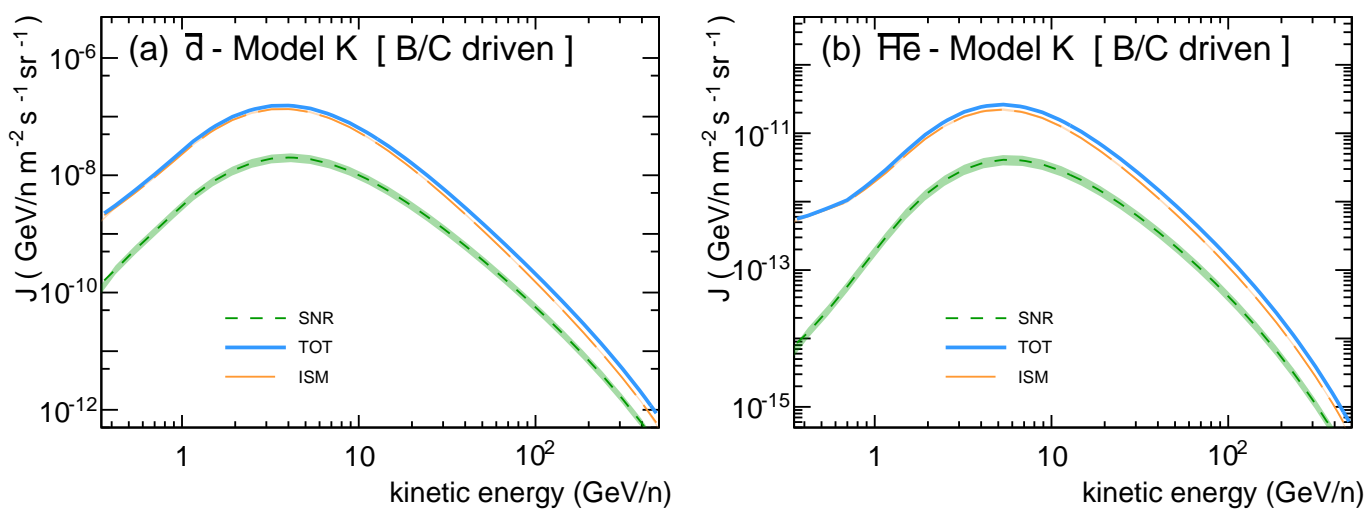

Figure 2: Model predictions of for the total flux of antideuterons (a) and antihelium (b), including secondary production inside SNRs (green short-dashed lines), standard production in the ISM (orange long-dashed lines), and total flux (lightblue solid lines). Calculations are fromx the B/C-driven parameter setting.
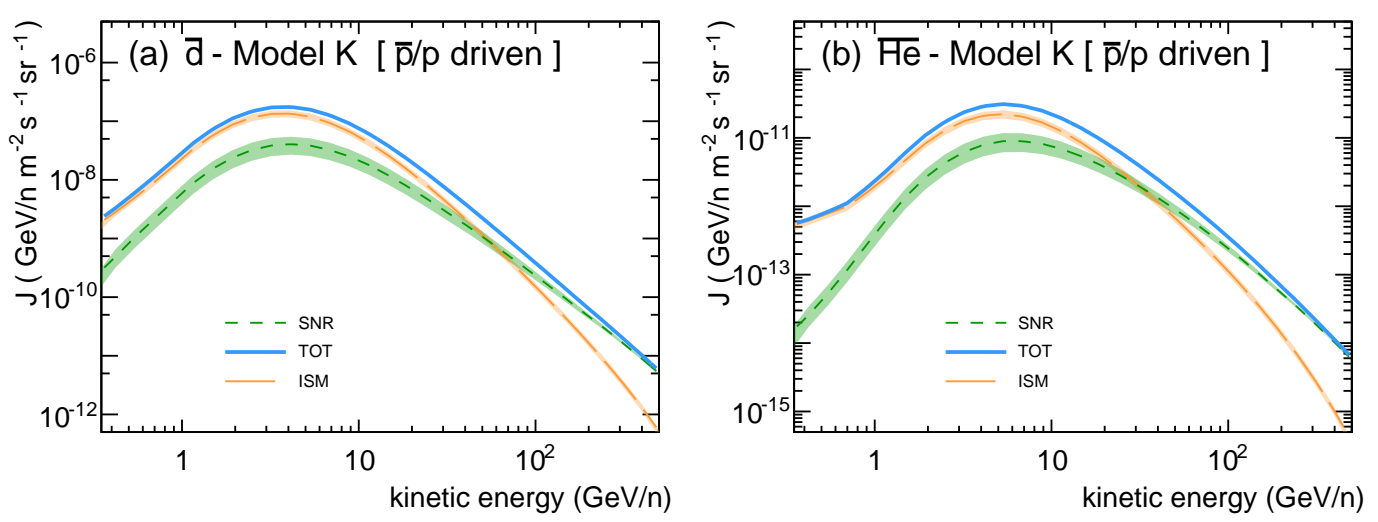

Figure 3: Model predictions of for the total flux of antideuterons (a) and antihelium (b), including secondary production inside SNRs (green short-dashed lines), standard production in the ISM (orange long-dashed lines), and total flux (lightblue solid lines). Calculations are fromx the B/C-driven parameter setting.

respectively. It can be seen from the figure that the SNR flux contribution is always sub-dominant over the considered energy range $0.5-500 \mathrm{GeV} / \mathrm{n}$. Under this model, however, SNR-accelerated secondaries do not appreciably contribute to the total flux in low-energy region, although tertiary production processes have been included in the calculations. In Fig. 3, flux calculations of both species are presented for the $\bar{p} / p$-driven parameter settings. In contrast to the more conventional B/C-driven approach of Fig. $2, \bar{p} / p$-driven predictions give an enhanced flux of SNR-accelerated antinuclei. We also note that the $\bar{p} / p$ ratio is more tightly connected with the $\overline{\mathrm{d}}$ and $\overline{\mathrm{He}}$ spectra, because all these antiparticles $(\bar{p}, \overline{\mathrm{d}}, \overline{\mathrm{He}})$ are generated from the fragmentation of the same $\mathrm{CR}$ species.

From calculations shown in Fig. 3, it can be noticed that the SNR-accelerated fluxes become dominating in the energy region above $\sim 100 \mathrm{GeV} / \mathrm{n}$. At the energy of about $\sim 500 \mathrm{GeV} / \mathrm{n}$, the total fluxes of $\overline{\mathrm{d}}$ and $\overline{\mathrm{He}}$ are one order of magnitude larger than those arising from B/C-driven calculations. In such a high-energy region the total flux is expected be very weak, at the level of $10^{-11}$ $\left(10^{-14}\right) \mathrm{GeV}^{-1} \mathrm{~m}^{-2-1} \mathrm{sr}^{-1}$ for $\overline{\mathrm{d}}(\overline{\mathrm{He}})$ particle. This level is inaccessible by the existing CR detec- 
tion experiments. On the other hand, in the sub-GeV/n energy region, $\bar{p} / p$-driven calculations of Fig. 3 do not shown substantial differences in comparison with the B/C-driven results of Fig. 2. At these energies, hadronic production processes in SNRs do not provoke significant modifications on the expected fluxes. Furthermore, in spite of appreciable error bands on the SNR and ISM component, the total ISM+SNR flux prediction is found to be rather stable for a large region of parameter space. $\mathrm{B} / \mathrm{C}$ and $\bar{p} / p$-driven prediction give both consistent results for the total flux intensity of CR antinuclei in the $\mathrm{GeV} / \mathrm{n}$ energy region, event though the single SNR and ISM contributions appear to be substantially different. In this respect, the total astrophysical background for DM searches appears to be soundly assessed at mid-low energies. These consideration do not apply to the shape of DM induced signals. The spectra of antinuclei produced from DM annihilations, in fact, suffer from different types of parameter degeneracy because the DM source is expected to be distributed in the whole Galactic halo. Thus, when modeling DM-induced CR particles, constaints provided by the $\mathrm{B} / \mathrm{C}$ ratio are not sufficient to characterize their Galactic transport. We also recall that the antinuclei flux calculations are affected by large nuclear uncertainties that we have not addressed in this work. These uncertainties have a similar influence on ISM and SNR components, being the two contributions tightly correlated each other.

\section{Conclusions}

This work is motivated by the search for a model of origin and propagation of Galactic CRs that is able to account for the several conflicting observations in their energy spectrum. SNRs are thought to be the main sources of primary CRs in the Galaxy such as proton, He, or C-N-O nuclei. In this work, we have used new data from AMS to determine the effect of production and acceleration of secondary CR particles inside these sources. Consequence of this mechanism is a progressive flattening of secondary-to-primary ratios at high energies. We have tested this mechanism under a two-halo scenario of CR propagation, based on Kolmogorov diffusion in the Galactic disk, which provides an accurate description of the primary CR spectra. We found in the first place that the AMS data support the production of secondaries in SNRs. Accounting for such processes, in fact, provides a good description of the $\mathrm{B} / \mathrm{C}$ data in the $\mathrm{GeV}-\mathrm{TeV}$ range. Furthermore, the resulting amount of B-nuclei produced by inside SNRs appears to be in line with naive expectations, as noted in [35]. Unfortunately, this mechanisms introduces new degeneracies between acceleration and transport parameters. Our fits to the $\bar{p} / p$ ratio data from AMS provides further evidence for secondary production in SNRs, but the $\bar{p} / p$-driven parameters are found to be inconsistent at $95 \% \mathrm{CL}$ with the $\mathrm{B} / \mathrm{C}$-driven paramters. Hence, the tension between $\mathrm{B} / \mathrm{C}$ and $\bar{p} / p$ ratios, already noted within conventional models of $\mathrm{CR}$ propagation, cannot be resolved in terms of hadronic interaction inside CR accelerators.

With the constraints provided by the two ratios, we have presented calculations for the expected fluxes of $\overline{\mathrm{d}}$ and $\overline{\mathrm{He}}$ antinuclei in CRs. As we have shown, the SNR flux component of CR antinuclei can be appreciably large at high energy. However, in the low-energy region between $\sim 0.1$ and a few $\mathrm{GeV} / \mathrm{n}$, which is where DM-induced signatures have chances to exceed over the background, the SNR-accelerated flux is found to be always sub-dominant. Furthermore, we found that the total ISM+SNR flux of antinuclei is rather stable for a large region of parameter configurations, event though the single SNR and ISM contributions appear to be substantially different and 
highly model-dependent. Thus the astrophysical background for DM searches in the low-energy region appear to be soundly assessed in this energy region. The antideuteron and antihelium search is ongoing AMS experiment, and soon, by the GAPS detection project [17].

We thank our colleagues of the AMS Collaboration for valuable discussions. NT acknowledges the European Commission for support under the H2020-MSCA-IF-2015 action, grant agreement No.707543-MAtISSE. AO acknowledges CIEMAT, CDTI and SEIDI MINECO under grants ESP2015-71662-C2-(1-P) and MDM-2015-0509.

\section{References}

[1] T. Aramaki, et alii, Phys. Rept. 618, 1-38 (2016); L. A. Dal, \& M. Kachelrieß, Phys. Rev. D 86, 103536 (2012); A. Ibarra, \& S. Wild, Phys. Rev. D 88, 023014 (2013); L. A. Dal, \& A. R. Raklev, Phys. Rev. D 91, 123536 (2015)

[2] M. Cirelli, et alii, JHEP 08, 009 (2014); E. Carlson, et alii, Phys. Rev. D 89, 076005 (2014); K. Blum, et alii, arXiv:1704.05431 (2017)

[3] N. Fornengo, Proc. XXV ECRS-2016, Torino, [arXiv:1701.00119] (2017)

[4] M. Aguilar, et alii, Phys. Rev. Lett. 117, 091103 (2016)

[5] P. Salati, Proc. 17 $7^{\text {th }}$ Lomonosov Conf. 2015, Moscow [arXiv:1605.01218] (2016)

[6] P. Lipari, Phys. Rev. D 95, 063009 (2017)

[7] K. Kohri, et alii, Prog. Theor. Exp. Phys. 021E01 (2016)

[8] J. Feng, N. Tomassetti, A. Oliva, Phys. Rev. D 94, 123007 (2016)

[9] G. Giesen, et alii, JCAP 09, 23 (2015); C. Evoli, D. Gaggero, D. Grasso, JCAP 12, 039 (2015); R. Kappl, A. Reinert, M. W. Winkler, JCAP 10, 34 (2015);

[10] C. H. Chen, C. W. Chiang, T. Nomura, Phys. Lett. B 747, 495-499 (2015); K. Hamaguchi, T. Moroia, K. Nakayama, Phys. Lett. B 747, 523-528 (2015) B. Q. Lu, \& H. S. Zong, Phys. Rev. D 93, 103517 (2016); A. Cuoco, M. Kramer, M. Korsmeier, Phys. Rev. Lett. 118, 191102 (2017); M. Y. Cui, et alii, Phys. Rev. Lett. 118, 191101 (2017)

[11] P. Blasi, \& P. D. Serpico, Phys. Rev. Lett. 103, 081103 (2009); Blasi, P., Phys. Rev. Lett. 103, 051104 (2009)

[12] P. Mertsch, \& S. Sarkar, Phys. Rev. D 90, 061301 (2014); P. Mertsch, \& S. Sarkar, Phys. Rev. Lett. 103, 081104 (2009)

[13] N. Tomassetti, \& J. Feng, Astrophys. J. 835, L26 (2017)

[14] J. Herms, et alii, JCAP 02, 018 (2017)

[15] I. Cholis, D. Hooper, T. Linden, [arXiv:1701.04406] (2017)

[16] A. Kounine, Proc. 32 ${ }^{\text {nd }}$ ICRC - Beijing, China, 12, 5-12 (2011)

[17] T. Aramaki, et alii, Astropart. Phys. 74, 6 (2016)

[18] A. Coogan \& S. Profumo, [arXiv:1705.09664] (2017); J. Sokol, Science, April 4 ${ }^{\text {th }}, 2017$ [ScienceMag] 
[19] I. A. Grenier, J. H. Black, A. W. Strong, Annu. Rev. Astron. Astrophys., 53, 199âĂŞ246 (2015); E. Amato \& P. Blasi, Adv. Space Res., in press (2017) [arXiv:1704.05696]; P. Blasi, Astron. Astrophys. Rev. 21, 70 (2013)

[20] N. Tomassetti, Astrophys. Space Sci. 342, 131-136 (2012)

[21] N. Tomassetti, Phys. Rev. C 92, 045808 (2015)

[22] M. di Mauro, et alii, Phys. Rev. D 90, 085017 (2014)

[23] J. R. Letaw, R. Silberberg, C. H. Tsao, Astrophys. J. Suppl. Series 51, 271-275 (1983); R. K. Tripathi, M. G. Cucinotta, J. W. Wilson, Nucl. Inst. Meth. B 117, 347-349 (1996)

[24] B. Alper, et alii, Phys.Lett. 47B (1973) 275-280; W. M. Gibson, et alii, Lett. Nuovo Cim. 21, 189 (1978)

[25] F. Donato, N. Fornengo, D. Maurin, Phys. Rev. D 78, 043506 (2008); P. Chardonnet, J. Orloff, P. Salati, Phys. Lett. B 409, 3, 13-320 (1997) R. Duperray, et alii, Phys. Rev. D 71, 083013 (2005)

[26] H. G. Fisher, APH N.S., Heavy Ion Phys. 17, 369-386 (2003)

[27] K. C. Tan, \& L. K. Ng, J. Phys. G: Nucl. Phys. 9, 227 (1983)

[28] I. Cholis, \& D. Hooper, Phys. Rev. D 89, 043013 (2014)

[29] N. Tomassetti \& F. Donato, Astron. \& Astrophys. 544, A16 (2012)

[30] N. Tomassetti, Phys. Rev. D 92, 081301 (2015); N. Tomassetti, Astrophys. J. 752, L13 (2012);

[31] Y. Q. Guo, Z. Tian, C. Jin, Astrophys. J. 819, 1 (2016); Y. Q. Guo, \& Q. Yuan, [arXiv:1701.07136] (2017)

[32] A. Ghelfi, et alii, Adv. Space Res. (2017) [arXiv:1607.01976]; N. Tomassetti, Adv. Space Res. (2017) [arXiv:1610.06187]

[33] R. Kappl, Comp. Phys. Comm. 207, 386-399 (2016)

[34] M. Aguilar, et alii, Phys. Rev. Lett. 117, 231102 (2016)

[35] R. Aloisio, P. Blasi, P. D. Serpico, Astron. \& Astrophys. 583, A95 (2015); P. Blasi, E. Amato, P. D. Serpico, Phys. Rev. Lett. 109, 061101 (2012)

[36] N. Tomassetti \& F. Donato, Astrophys. J. 803, L15 (2015)

[37] N. Tomassetti, Astrophys. J. 815, L1 (2015); N. Tomassetti, Phys. Rev. D 92, 063001 (2015); M. Kachelrieß, A. Neronov, D. V. Semikoz, Phys. Rev. Lett. 115, 181103 (2015);

[38] A. D. Erlykin, \& A. W. Wolfendale, Astropart. Phys. 35, 449-456 (2012); S. Thoudam, \& J. Horandel, MNRAS 435, 2532 (2013); Y. Y. Keum, \& P. Salati, Pramana 86-2, 369-377 (2016) 